\title{
Teach your meetings well Backward design for better virtual meetings
}

A s COVID-19 continues to influence the ways in which academic libraries serve faculty and students, so too does it impact the internal communications, planning, and processes of library teams. As libraries adapt to fully remote or hybrid staffing arrangements, meetings have shifted to platforms such as Zoom, WebEx, and Microsoft Teams. Flexible work arrangements, in which some staff may be onsite while others are dispersed, are likely to persist even as campuses return to more face-to-face operations. Leading effective meetings in person is a learned skill, one that can be adapted to the remote environment with some help from best practices in digital pedagogy and, in particular, the principles of backward design. These best practices can improve meeting productivity, encourage interaction, and increase inclusion for colleagues in satellite locations.

\section{Background}

There is no shortage of literature on the characteristics of effective meetings. Sharing an agenda, keeping minutes, respecting the time of participants, staying on task, maintaining an appropriate environment, and encouraging participation are commonly accepted practices for success. ${ }^{1}$ Many library practitioners have a great deal of experience leading meetings in person, but less so leading meetings in a dispersed environment. Encouraging and managing participation, for example, requires a different approach on Zoom, as does maintaining an appropriate meeting environment. Even with cameras on, it can be difficult to read the social and nonverbal cues that are typically critical for effective communication. ${ }^{2}$ Indeed, creating a sense of psychological safety, described as "people feeling they can raise ques- tions, concerns, and ideas without fear of personal repercussion,"3 presents a new challenge in remote environments, especially for new teams that may not already have trusting relationships established. Much like a virtual classroom, the most effective online meetings require skilled facilitation and full engagement by participants.

When done well, virtual meetings have the potential to be even more effective than in-person meetings. They may provide the facilitator with a greater degree of control, speed, structure, and clarity, especially for activities such as brainstorming. ${ }^{4}$ They also mitigate the inherent imbalance in meetings in which some participants are literally "in the room" while others find themselves listening in remotely. Hybrid meetings require particular attention to ensure that in-person participants do not dominate the conversation to the exclusion of remote participants. ${ }^{5}$ To capitalize on the benefits of virtual meetings and mitigate potential problems, it is helpful to reflect on successful approaches in remote instruction.

Remote teaching has its own best practices rooted in instructional design. Instructional design models can effectively organize and structure content into learner-centered lesson plans for online instruction, while incorporating the added variable of web conferencing technology. Backward design is one such approach. This model asks instructors to 1) identify goals and desired results, 2) determine

Rachel G. Rubin is associate university librarian for research and learning, email: rachel.rubin@pitt.edu, and Urszula Lechtenberg is learning design coordinator, email: url1@pitt.edu at the University of Pittsburgh University Library System 
acceptable evidence of meeting those goals, and 3) design learning experiences and instruction based on that information. ${ }^{6}$ In a technologically dependent setting, defining outcomes and desired results requires that the impact of instructional technologies be considered: whiteboards, breakout rooms, and other tools embedded in web conferencing platforms can be implemented in support of reaching intended goals.

Matching instructional technologies with learning (or meeting) scenarios can be both exciting and frustrating, considering the number of tools available and the amount of time it might take to learn those tools well. Facilitators can start with the technologies provided and supported by their organization (e.g., LibGuides, LibWizard, or Zoom breakout rooms) and match them to their intended goals. While it is impossible to become an expert in all available platforms, Char Booth provides a useful set of steps that help balance maintaining current awareness of technologies while adapting to constant change. 1) Experience: authentic, hands-on experiences will demystify a platform or tool. Try testing it out in a low-stakes environment with colleagues before using it in practice. 2) Evaluate: in line with backward design, consider if and how the technology will achieve specific outcomes. 3) Customize: incorporate the technology based on the actual needs and objectives of your teaching or meeting scenario. ${ }^{7}$ Through this strategy, instructors and meeting facilitators can build their toolkits to design effective experiences using various technologies and become sufficiently comfortable with a few key platforms.

\section{Strategies}

Most meetings do not require (nor do most employees have the time to devote to) rigorous design and assessment. No matter the importance of the meeting, however, it is worthwhile to spend a little time revisiting these backward design principles when creating your agenda: 1) identify goals and desired results, 2) consider what would constitute evidence of meeting the goals, and 3) design the meeting experience to ensure success and inclusion. With practice, these steps can become a relatively quick and efficient way of adapting teaching and learning approaches to effective meetings. If a topic is worth meet- ing about, it is also worth investing some effort to ensure that the meeting is a good use of everyone's time.

\section{Identify goals and desired results}

The first step is to identify the overall purpose of the meeting and the goal of each agenda item. Is the meeting intended to generate discussion, share information, or to make decisions? Is it for big-picture brainstorming, gathering feedback, or project planning? It is not unusual for meetings to address more than one objective, so in addition to thinking about the overall purpose, it is important to reflect on the intended outcome of each agenda item. Next, consider what resources (people, documents, or supplementary information, for example) will be necessary to successfully meet each goal. Recurring project or deadlinebased meetings may benefit from this type of planning. Working backward from a deadline or intended outcome, for example, meetings can be scaffolded to build up to the completed project by working through the purpose, goals, and desired results for each meeting in advance. These preliminary steps will also help to select appropriate interactive technologies later in the process.

\section{Identify evidence of meeting the goals}

Agenda items may result in immediate outcomes such as decisions or actions, but everyone has experienced "next step limbo," especially when goals are unclear. One way to quickly evaluate if the goal has been achieved is to create a standard nomenclature for desired outcomes that can be applied to each agenda item. For example, each topic can be clearly labeled discussion, action item, decision, or announcement on the agenda itself. This process requires that the person creating the agenda take the time to identify what form "success" will take. For example, consider the agenda item "continuing no-contact pickup." The subject is clear, but the goal is not. Is the purpose to make a decision, to have a discussion, or to share an announcement? Alternatively, the agenda item could read: "Decision: continuing no-contact pickup." Simply by adding a notation about the purpose of the item, two needs are met: 1) participants understand the expectations for their participation on that item prior to the meet- 
ing, and 2) it is easy to assess whether the goal has been met. Was a decision made or not? In this case, the "evidence" is not tied to what happens after or outside the meeting. Here, success is measured by whether the stated expectation for the item was met during the meeting: yes, a decision was made.

\section{Design the meeting experience}

Design and implementation are where in-person and virtual experiences diverge most. While purpose and goals may be shared, technology adds an extra layer of complexity to the facilitation and engagement process. This is in part because a meeting must function on multiple levels. In an online environment the existence of those levels can be more palpable because they require more intention and attention: facilitators need to be able to select and be comfortable using the appropriate tools (e.g., whiteboard, chat, polls); interpersonal dynamics must be managed while navigating mixed use of cameras, sound or visual delays, and diverse levels of technological prowess; and last, but certainly not least, the meeting itself must stay on time and on task. One way to approach this challenge is to break down the design phase into tools and rules.

The first step is to match the appropriate tools to the objectives of the meeting. For a highly interactive activity such as brainstorming, for example, one approach is to share your screen and capture contributed ideas in real time. This can be done by the facilitator or an assigned notetaker in a shared document. This ensures that all participants can see and build on previous comments, correct any misrepresentations or clarify confusion, and engage in creative thinking without worrying about taking notes. An integrated whiteboard can also be used effectively for this.

If the goal is decision making, tools like polling and screen annotations may be useful. The polling option in Zoom allows the host to create a set of multiple-choice questions that can be shared at any point of the meeting. Once answers are selected, the votes are tallied, and the results are displayed. A benefit of this approach is anonymity: a poll might be the best way to elicit honest feedback if the topic is sensitive or if a team has not yet developed a high degree of trust. For a more interactive approach, consider using screen annotations. As a meeting host in Zoom, you can share the topics or objects under consideration, whether they are on a website, in a shared document, or even on a whiteboard. Participants can then mark their choices using the annotation feature, which includes checkmarks, stars, and hearts.

Lastly, many meetings consist of discussion items. For this type of activity, breakout rooms can be a good tool to organize larger groups for discussion. Participants can be populated into small groups randomly or in advance. In Zoom, the facilitator can also create rooms based on topics, themes, or questions, and participants can self-select their discussion forum. Discussion-heavy meetings can be particularly difficult to manage in the online setting, which is one reason why having clear rules is so important.

Regardless of the tools employed, shared guidelines for participation are essential to ensure equitable opportunities for engagement. For example, you might require that participants use visual cues, such as a raised hand, to indicate a desire to speak. Such a system can be tailored so that a raised hand might signify a comment on a new subject, while a green "yes" checkmark indicates a follow-up comment on the current topic. ${ }^{8}$ A moderated system such as this can prevent individuals from dominating the conversation, takes the burden off speakers to "jump in," allows participants to focus on listening and speaking in an orderly fashion, and gives the facilitator the ability to manage the conversation while mitigating a lack of social cuing. Rules for when to use the chat function may also come into play, especially if not all participants have access to a microphone. No matter what rules the facilitator and group members establish, everyone must agree to follow them so that participants can focus on what they want to say, not how they will be able to say it.

\section{Conclusion}

Virtual meetings can be socially complex and technologically challenging. The virtual environment can make it difficult to read social cues that normally facilitate in-person discussion, and mixed access to and comfort with technology can be a barrier. For meeting facilitators, the pressure to be comfortable with many technologies can feel overwhelming. The best practices discussed here can be applied with varying degrees of rigor to 
meetings of all types to help reduce these stresses. Taking a backward design approach has the potential to make virtual meetings equally if not more inclusive, efficient, and productive than in-person meetings. As academic libraries continue to integrate the lessons learned during the pandemic, the benefits of virtual meetings can be sustained. With some direction from backward design and a bit of planning up front, future meetings may be even more fruitful and productive meeting experiences for all participants.

\section{Notes}

1. Linda A LeBlanc and Melissa R. Nosik, "Planning and Leading Effective Meetings," Behavior Analysis in Practice 12 no. 3 (2019): 696-708.

2. Amy C. Edmonson and Gene Daley, "How to Foster Psychological Safety in Virtual Meetings," Harvard Business Review Digital Articles, August 25, 2020: 2-5, https://hbr.org/2020/08 /how-to-foster-psychological-safety-in-virtual -meetings.
3. Edmonson and Daley, "How to Foster," 2.

4. Bob Frisch and Cary Greene, "3 Things Virtual Meetings Offer that In-Person Ones Don't," Harvard Business Review Digital Articles, July 23, 2020: 2-9, https://hbr.org/2020/08 /how-to-foster-psychological-safety-in-virtual -meetings.

5. LeBlanc and Nosik, "Planning and Leading."

6. Grant Wiggins and Jay McTighe, Understanding by Design (Expanded 2nd ed.) (Alexandria, VA: Association for Supervision and Curriculum Development, 2005).

7. Char Booth, Reflective Teaching, Effective Learning: Instructional Literacy for Library Educators (Chicago: American Library Association, 2011), 64.

8. Kate Joranson and Alison Langmead, "Inclusive Goals and Strategies for Co-Learning Using Video Conferencing" Co-Learning Encounters, June 10, 2020, https://colearning.hcommons. org/2020/06/10/inclusive-goals-and-strategies -for-co-learning-using-video-conferencing/. z
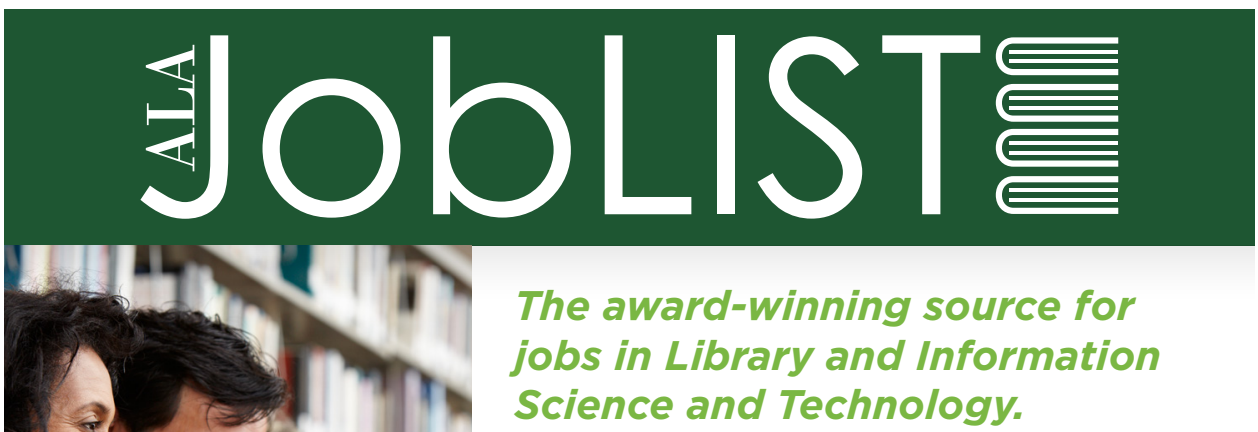

JOB SEEKERS

Filter and sort hundreds of job ads by position type, employer, location, job title, and more.

\section{EMPLOYERS}

Strengthen your candidate pool-ALA reaches the engaged professionals and students you want to hire.

\section{joblist.ala.org}

\section{ALA Arater Library

